\title{
ADAM12 as a Clinical Prognostic Indicator Associated with Tumour Immune Infiltration in Lung Adenocarcinoma
}

Junfan Pan

Fujian Medical University

Zhidong Huang

Fujian Medical University

Yiquan Xu ( $\nabla$ xuyiquan1018@fjmu.edu.cn )

Fujian Medical University https://orcid.org/0000-0003-4189-9692

\section{Primary research}

Keywords: ADAM12, lung adenocarcinoma, prognostic indicator, tumour immune microenvironment

Posted Date: May 3rd, 2021

DOI: https://doi.org/10.21203/rs.3.rs-452333/v1

License: (c) (i) This work is licensed under a Creative Commons Attribution 4.0 International License.

Read Full License 


\section{Abstract}

Background: Lung cancer is the most common cause of cancer-related death worldwide. In humans, 22 functional a-disintegrinand metalloproteinases (ADAMs) have been identified, 12 of which have proteolytic activity. The role of ADAMs in cancer has attracted increasing attention. However, the expression and significance of ADAMs in lung adenocarcinoma (LUAD) remain unclear. The current study aimed to explore the expression and prognostic value of ADAM12 in LUAD.

Methods: The Cancer Genome Atlas(TCGA) database was used to analyse the expression of ADAMs in LUAD. The cBioPortal database was used to obtain and analyse ADAM12 copy number changes, and the LinkedOmics database was utilised for the analysis of ADAM12-related genes. Kyoto Encyclopedia of Genes and Genomes (KEGG) enrichment analysis was then performed using TIMER. The relationship between ADAM12 and the tumour immune microenvironment(TIM) was assessed via the TISIDB database. ADAM12 and immune-related genes were used to construct a prognostic model. Knockdown of ADAM12 was performed in vitro in order to verify its biological function. The unpaired $t$-test was used for comparison between the two groups, ANOVA was used for analysing differences between multiple groups, and the Kaplan-Meier test was used to compare survival between groups.

Results: Most ADAMs exhibited significant differential expression in LUAD. ADAM12 expression was significantly higher in LUAD tissues than in healthy tissues, and lower ADAM12 expression was associated with better survival. Genetic alterations of ADAM12 mainly included missense mutations, amplifications, and deep deletions. ADAM12 and positively correlated genes were mainly enriched in protein digestion and absorption, ECM-receptor interaction, and adhesion plaques. ADAM12 had a moderate correlation with immune cell markers EBIP1, CCNB1, EX01, KNTC1, PRC1 and FAM198B. Prognostic model was established based on ADAM12 and immune-related genes. In vitro experiments revealed that knocking down ADAM12 inhibited cell proliferation, migration and invasion.

Conclusion: ADAM12 potentially plays an important role in the occurrence of LUAD and may be utilised as an immunotherapy target and a valuable prognostic biomarker for LUAD.

\section{Background}

Lung cancer is the most common cause of cancer-related death worldwide. Annually, 1.8 million new cases of lung cancer are diagnosed, and 1.6 million lung cancer patients die, of which approximately $85 \%$ suffer from non-small cell lung cancer (NSCLC) ${ }^{[1]}$. NSCLC mainly includes lung adenocarcinoma(LUAD) and lung squamous cell carcinoma(LUSC), with the former being most frequent ${ }^{[2,3]}$. LUAD is common among non-smokers, women and East Asian patients. Over the past 20 years, the introductions of targeted therapies and immunotherapy have benefited certain patients with advanced NSCLC. However, LUAD treatment remains challenging, necessitating the identification of new driver genes to expand the patient population benefiting from targeted therapy. Further, new predictors of immunotherapy response are also required. 
22 functional a-disintegrin and metalloproteinases (ADAMs) have been identified in humans, but only 12 of these have proteolytic enzyme activity (ADAM8, 9, 10, 12, 15, 17, 19, 20, 21, 28, 30 and 33) ${ }^{[4]}$. ADAMs are involved in a variety of biological processes, including cell adhesion, intercellular signal transduction, extracellular matrix dynamics, and cellular transport $[5,6]$. The role of ADAMs in cancer has attracted increasing attention. Studies have shown that ADAM8 upregulates miR-720 expression, which in turn promotes triple-negative breast cancer (TNBC) metastasis by regulating the $\beta 1$-integrin/ERK signalling pathway, and is thus expected to serve as a biomarker for the early detection of ADAM8-positive TNBC or a predictor of treatment response ${ }^{[7]}$. ADAM17 expression is generally high in gastric cancer, colon cancer, liver cancer, breast cancer, NSCLC, and other cancer types, promoting tumour metastasis through Notch and Wnt signalling [5, 8-11]. In lung cancer, ADAM9, ADAM19 and ADAM23 are highly expressed, whileADAM28 expression is low ${ }^{[12-15]}$. Studies have reported that in LUAD brain metastasis, ADAM9 promotes vascular remodelling via promoting the expression of vascular endothelial growth factor $A$ (VEGFA), angiopoietin 2 (ANGPT2) and tissue plasminogen activator (PLAT) expression, in turn compromising blood-brain barrier integrity ${ }^{[12]}$. ADAM12 is highly expressed in a variety of tumours, promoting invasion and metastasis ${ }^{[16-18]}$. Bioinformatics analysis of LUAD previously revealed that the high expression of ADAM12, BTK and ERG is mainly related to adhesion plaques and the activation of immune modulatory pathways ${ }^{[19]}$. However, no reports have focused on ADAM12 in LUAD. In recent years, a great number of studies have demonstrated that the tumour immune microenvironment (TIM) plays a vital role in cancer progression and therapeutic efficacy. The tumour microenvironment (TME) generally consists of tumour cells, immune cells and non-immune cell subsets (e.g. fibroblasts, pericytes, endothelial cells) as well as extracellular matrix (ECM) proteins, cytokines, and growth factors ${ }^{[20]}$. ADAMs belong to the superfamily of zinc-dependent metalloproteinases, which process and degrade ECM proteins, growth factor-binding proteins, cell adhesion molecules as well as cell surface receptors ${ }^{[21,22]}$. Therefore, ADAM shave major implications within the TME. In a variety of cancers, matrix metalloproteinases (MMPs) and ADAMs cleave the NKG2D ligand from the surface of tumour cells, preventing its interaction with the NKG2D receptor on the surface of NK cells. As a result, the receptor

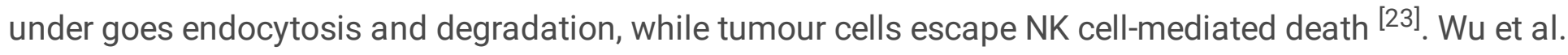
found that, within the TME, ADAM17 specifically cleaves activated FcyR CD16A on NK cells, causing them to degranulate, which in turn compromises their tumour-killing effect ${ }^{[24]}$. This observation suggests that ADAM17 may be a potential target for cancer immunotherapy. Taken together, it is essential to further our understanding of the role of ADAMs within NSCLC. In particular, the function of ADAM12 within the TIM has attracted our attention.

In this study, we obtained The Cancer Genome Atlas(TCGA)data to conduct an in-depth analysis of 12 ADAMs with proteolytic enzyme activity in LUAD tissues and adjacent normal tissues. More specifically, we sought to explore differentially expressed ADAM-encoding genes in LUAD, subsequently focusing on ADAM12. We then assessed its association with clinical pathological characteristics, patient survival and the TIM, thus providing guidance for LUAD treatment. 


\section{Methods}

\section{Data acquisition}

\section{Identification of differentially expressed ADAM-encoding genes in TCGA data}

In this study, TCGA (http://cancergenome.nih.gov/) RNA-seq data from a total of 551 LUAD patients were used, including 497 LUAD tissues and 54 healthy lung tissues. Twelve ADAMs were analysed, including ADAM8, 9, 10, 12, 15, 17, 19, 20, 21, 28, 30 and 33.The limma software package in $R$ version (4.0.2) was used to identify ADAMs differentially expressed between tumour and healthy tissue. Genes with a $p$ value $<0.05$ and $\mid \log 2$ (fold change)|>1 were considered to have significant differential expression. Subsequently, heatmaps were constructed to visualise the differential expression of ADAMs between the two groups.

\section{cBioPortal database analysis}

The cBioPortal Cancer Genomics Portal (http://cbioportal.org) is an open resource with data from 225 cancer studies $^{[25]}$. It was used to analyse the frequency of ADAM12 genetic alterations in LUAD (including mutations, deletions and copy number variation). All searches were performed according to the instructions provided on the cBioPortal website.

\section{LinkedOmics database analysis}

LinkedOmics (http://www.linkedomics.org/login.php) is an online tool used for the analysis of TCGA data ${ }^{[26]}$.We used the LinkFinder module and Spearman correlation analysis to determine genes related to ADAM12 in LUAD. We then built a heatmap of co-expressed genes. After obtaining the strongly correlated co-expressed genes, we used R software to perform Kyoto Encyclopedia of Genes and Genomes (KEGG) enrichment analysis of these genes.

\section{Immune infiltration database (TIMER and TISIDB)}

We used the TIMER (https://cistrome.shinyapps.io/timer/) to analyse the correlation between ADAM12 expression and the infiltration of six major immune cell types, as well as to explore the relationship between the expression of ADAM12 and immune cell markers. We used the TISIDB database (http://cis.hku.hk/TISIDB/) to assess whether ADAM12 was related to LUAD immunotherapy outcome and immune cell subtypes, with the aim to elucidate the interaction between ADAM12 and tumour immunity.

\section{Construction of a prognostic risk score model}

We sought to develop a prognostic model of multiple ADAM12-related immune genes. We performed univariate Cox regression analysis, and used the Least Absolute Contraction and Selection Operator 
(LASSO) Cox regression algorithm to build a prognostic model based on the expression of selected genes. The risk score of the predictive model was calculated as follows:

risk score $=$ coefficient $1 \times$ value $1+$ coefficient $\mathrm{n} \times$ value $\mathrm{n}$,

where the value is the relative expression of each selected gene's z-score conversion. This formula was applied to calculate the risk score for each patient in TCGA database.

\section{Cell culture and cell transfections}

Human bronchial epithelial cells BEAS-2B and the human LUAD cell line A549 were donated by Professor Huang Yi from Fujian Provincial Hospital. Human LUAD cell lines PC-9『H827 and H1299 were purchased from Central South University. All cells were cultured in RPMI 1640 medium (Gibco, USA) supplemented with $10 \%$ foetal bovine serum ( $10 \% \mathrm{FBS}$ ) under a humidified atmosphere of $37^{\circ} \mathrm{C}$ and $5 \% \mathrm{CO}_{2}$. Human ADAM12 targeted small interfering RNAs (siRNAs) was designed and purchased from hippobiotec (Huzhou, China) and Lipofectamine 2000 reagent (Thermo Fisher Scientific, USA) used for small RNA transfection. The siRNA sequence used in this study was as follows: ADAM12si\#1:5'-

CCUCAAGGCAACUAAGUAUdTdT-3';ADAM12si\#2:5'-GCGAGAUGAGAGAUGCUAAdTdT-3';ADAM12si\#3:5'GCAGAUAACCAAGGUUUAAdTdT-3';sicontrol:5'-UUCUCCGAACGUGUCACGUdTdT-3'.

\section{Quantitative reverse transcription-polymerase chain reaction (qRT-PCR)}

TRIzol reagent ( Invitrogen, USA) was used to isolate total cellular RNA. Reverse transcription of ADAM12 was performed using the PrimeScript ${ }^{\text {TM }}$ RT reagent Kit ( Takara, Japan). qRT-PCR was performed using the GoTaq® qPCR Master Mix kit (Promega, USA). ADAM12 expression in NSCLC cell lines was determined via the $2^{-\triangle \triangle C T}$ method. GAPDH expression was used as an internal control. The primer sequences used in this study were as follows: ADAM12, 5'-ATTCAGCAGTCAGTCTCAGCAC-3' (forward) and 5'CTTTTCAGCTTCTTCGCTGG 3'(reverse); GAPDH, 5'-GGTGTGAACCATGAGAAGTATGA-3' (forward) and 5'GAGTCCTTCCACGATACCAAAG 3'(reverse).

\section{Western blotting}

Cells were lysed with RIPA buffer (Beyotime) containing a protease inhibitor cocktail ( Thermo Scientific, Waltham, MA, USA) and PMSF. The protein concentration of samples was determined via the BCA method. 30-50 $\mu \mathrm{g}$ cell lysate was loaded and separated via SDS-PAGE. Protein was then transferred onto PVDF membranes ( Sigma, St. Louis, MO, USA), which were incubated with a specific primary antibody. They were then incubated with specific secondary antibodies. Autoradiography was employed for band density quantification ( Quantity One software; Bio-Rad). $\beta$-tubulin served as a loading control. The antibodies used were as follows: ADAM12 ( sc-293225, Santa Cruz, UA) and $\beta$-tubulin ( YM3139, Immunoway, China).

\section{Cell colony formation assay}


Cells were seeded in a 6-well plate ( 500 cells/well) and cultured with 10\% FBS RPMI 1640 medium for 10 days. The excess liquid was removed, cells were fixed with formaldehyde, stained with crystal violet ( Sigma) and placed on an IX71 inverted microscope ( Olympus, Tokyo, Japan). Images were then obtained, and cells were counted. All experiments were repeated at least three times.

\section{Wound-healing assay}

For the wound-healing experiment, cells were seeded into a 6 -well plate $\left(1 \times 10^{5}\right.$ cells/well), and a single layer of cells was scratched with a $10 \mu \mathrm{L}$ plastic pipette tip to form a uniform wound. The distance between the two edges of migrating cell sheets was photographed under a microscope and quantified. All experiments were repeated at least three times.

\section{Transwell migration and invasion assays}

For the migration assay, $2 \times 10^{4}$ cells in $200 \mu \mathrm{L}$ serum-free medium were placed in the upper chamber of the Transwell system ( $8 \mu \mathrm{m}$ pore size, BD Biosciences, San Jose, CA, USA). For the invasion assay, $5 \times$ $10^{4}$ cells in $200 \mu \mathrm{L}$ serum-free medium were transferred to the upper chamber (BD Biosciences) covered with Matrigel, and $600 \mu \mathrm{l}$ RPMI 1640 medium containing 10\% FBS was added to the lower chamber as a chemoattractant. After $24 \mathrm{~h}$ of incubation, the remaining cells in the upper chamber were removed, and those in the lower chamber were fixed with formaldehyde and stained with crystal violet ( Sigma). The cells were then imaged under an IX71 inverted microscope ( Olympus, Tokyo, Japan) and counted. All experiments were repeated at least three times.

\section{Statistical analysis}

TCGA data were analysed using R software (version 4.0.2), and all other experimental results were analysed using GraphPad Prism 8 statistical software. The unpaired $t$-test was used for comparison between the two groups, ANOVA was used for analysing differences between multiple groups, and the Kaplan-Meier test was used to compare survival between groups. For experiments performed in triplicate, the data are expressed as the mean \pm SD. $p<0.05$ indicated that the difference is statistically significant( ${ }^{*} p<0.05, * \star p<0.01$, and $\left.* * * p<0.001\right)$.

\section{Results}

\section{Abnormal expression of ADAMs in LUAD patients}

As some ADAMs play significant roles in proteolysis, we explored the expression levels of ADAMs (ADAM8, 9, 10, 12, 15, 17, 19, 20, 21, 28, 30 and 33) with proteolytic enzyme activity in LUAD. Most of these exhibited significant differential expression between LUAD and normal tissues (Fig. 1A, Table1). In particular, ADAM12, ADAM28, ADAM8, ADAM9, ADAM15, ADAM19 and ADAM20 expression was significantly inceased, whereas ADAM33, ADAM17 and ADAM10 expression was significantly decreased in LUAD tissues $(p<0.01)$. No significant differences were observed for ADAM30 and ADAM21 between 
the two groups. We then sought to clarify the correlation between ADAM expression. Correlation analysis revealed that ADAM19, ADAM12, ADAM 9, ADAM10 exhibited weak to strong correlations with other genes, among which ADAM 21 was most strongly correlated with ADAM $20(R=0.61)$, followed by ADAM 12 and ADAM 19 ( $R=0.5)$ (Fig. 1B). Taken together, correlation analysis indicated a certain association between the expression of different ADAMs. We further analysed the expression differences of ADAM12 between LUAD and normal tissues. ADAM12 expression was significantly increased in LUAD tissues. Further, low ADAM12 expression was associated with better survival (Fig. 1C, D).

\section{ADAM12 copy number changes altered its expression}

Considering that gene copy number changes are often pathogenic, we used the cBioPortal database to analyse ADAM12 copy number variation and found that ADAM12 genetic alterations mainly included mutations, amplifications and deep deletions. The change of copy number resulted in differential expression of ADAM12 mRNA ( Fig. 2A, B, C). To further understand the biological functions of ADAM12, we used the LinkedOmics database to analyse genes co-expressed with ADAM12 in LUAD. As shown in Figure 2D, 10,626 genes (red dots) exhibited a strong positive correlation with ADAM12, while 9,361 genes (green dots) had a strong negative correlation with ADAM12 (false discovery rate, FDR $<0.01$, |Spearman's correlation|>0.5). The top 50 positively correlated genes are shown in Figure 2E. Further, we conducted KEGG analysis of these 50 genes, which indicated that they were mainly enriched in protein digestion and absorption, ECM-receptor interactions and focal adhesions (Fig. 2F), suggesting ADAM12related genes were closely related to the TME.

\section{The influence of ADAM12 on the TIM}

As our findings suggested that ADAM12 is closely related to the $T M E$, we sought to explore whether ADAM12 is associated with tumour immune cell infiltration. We used gene set enrichment analysis (GSEA) to analyse abnormally activated signalling pathways related to ADAM12 differential expression (FDR<0.05, NOM $p$-value<0.05) (Fig. 3A). High expression of ADAM12 was closely related to ECM-receptor interactions, B cell receptor signalling pathways, NK cell-mediated cytotoxicity, T cell receptor signalling and Toll-like receptor signalling. Taken together, ADAM12 was closely related to tumour immune infiltration. TIMER data indicated changes in the copy number of multiple genes were related to the degree of immune cell infiltration. In particular, the chromosomal arm-level gain of ADAM12 was related to a decrease in B cell, $C D 4^{+}$cell and dendritic cell infiltration $(p<0.01)$. High amplification of ADAM12 was associated with lower infiltration of $\mathrm{CD}^{+}$cells and macrophages $(p<0.01)$ (Fig. 3B). Further, the expression level of ADAM12 was positively correlated with the degree of infiltration of most immune cell

types as well as with tumour purity $(p<0.05)$ (Fig. 3C). Based on available literature ${ }^{[27,28]}$, we selected $\mathrm{CD}^{+}{ }^{+}$cell, macrophage, neutrophil, and dendritic cell markers, in order to further explore the association of ADAM12 expression with the degree of immune cell infiltration. After tumour purification, cell markers with $|r|>0.4, p<0.001$ were screened. BRIP1 $(|r|=0.446)$, CCNB1 $(|r|=0.403)$, EX01 $(|r|=0.45)$, KNTC1 $(|r|=0.414)$, PRC1 $(|r|=0.418)$ and FAM198B $(|r|=0.423)$ had moderate to strong correlations with ADAM12 
(Fig. 3D, Table S1). Five of the six immune cell markers were CD4+T cell markers, suggestive of an important role for $\mathrm{CD} 4^{+} \mathrm{T}$ cells in the influence of ADAM12 on immune infiltration.

\section{The influence of ADAM12 and related immunomodulatory molecules on prognosis}

A variety of immune genes were found to be related to ADAM12 expression. Thus, we used the TISIDB database to further explore the correlation between the expression of immunomodulatory molecules and ADAM12. As shown in Figure 4A and B, most immunostimulatory and immunoinhibitory proteins were positively correlated with the expression of ADAM12. Among these, TNSF4 $(|r|=0.68, p<0.001)$ and PDCD1LG2 $(|r|=0.402, p<0.001)$ exhibited the strongest correlation with ADAM12. LUAD was divided into six immune-based categories, including $\mathrm{C} 1$ (wound-healing); C2 (IFN-gamma-dominant); C3 (inflammatory); C4 (lymphocyte-depleted); C5 (immunologically quiet); C6 (TGF- $\beta$-dominant). ADAM12 expression was highest in the TGF- $\beta$-dominant group, while the lymphocyte-depleted group exhibited the lowest ADAM12 expression level (Fig. 4C).In order to evaluate the impact of ADAM12 and related immunomodulators on prognosis, we established a LASSO regression model, using the curve of partial likelihood deviance versus log (lambda) to screen out six genes for the construction of a prognostic model (Fig. 5A,B and Table S2). Patients were then divided into high-risk and low-risk groups based on prognostic model risk score. The prognosis of patients was indeed more favourable in the low-risk group than in the high-risk group $(p<0.001)$ (Fig. 5C). To evaluate the accuracy of prognostic risk values, we performed ROC analysis. The AUC for 1-, 3-, and 5-year survival in TCGA data was $0.726,0.661$ and 0.639 , respectively (Fig 5D).We further analysed the relationship of high- and low-risk groups with immunoregulatory molecule expression as well as clinicopathological characteristics. Immunostimulatory CD40LG and CD80 expression as well as immunoinhibitory IL-10 expression was lower in the high-risk group. Further, the expression of ADAM12 as well as the immunostimulatory RAET1E and PVR was higher in the high-risk group. The $\mathrm{N}$ stage $(p<0.001), \mathrm{T}$ stage $(p<0.001)$, pathological stage $(p<0.001)$ and fustat $(p<0.001)$ were significantly different between the high- and lowrisk groups (Fig. 5E). We then employed univariate and multivariate Cox regression to analyse whether the risk score could be used as an independent prognostic factor. Univariate regression analysis indicated that stage $(\mathrm{HR}=1.646,95 \% \mathrm{Cl}=1.394-1.944, p<0.001), \mathrm{T}(\mathrm{HR}=1.597,95 \% \mathrm{Cl}=1.283-1.986, p<0.001), \mathrm{N}$ $(\mathrm{HR}=1.769,95 \% \mathrm{Cl}=1.440-2.174, p<0.001)$, and risk score $(\mathrm{HR}=5.563,95 \% \mathrm{Cl}=3.563-8.685), p<0.001)$ were significantly correlated with overall survival, while age, gender, and $\mathrm{M}$ stage were not (Fig. 5F).Multivariate Cox regression analysis suggested that risk score $(\mathrm{HR}=4.440,95 \% \mathrm{Cl}=2.657-7.418$, $p<0.001$ ) could be used as an independent prognostic factor (Fig. 5G). Taken together, these results indicated that ADAM12 and related immunomodulators have prognostic potential in LUAD patients.

\section{ADAM12 knockdown inhibited the proliferation, migration and invasion of LUAD cells in vitro}

We employed western blotting to detect ADAM12 protein expression in BEAS-2B and LUAD cells. ADAM12 expression was generally high in LUAD cells (Fig. 6A). We transfected siADAM12 into PC-9 cells and confirmed knockdown efficiency via qPCR (Fig. 6B). We then employed a cell colony formation assay to assess cell proliferation ability. ADAM12 knockdown significantly suppressed proliferation(Fig. 
6C). Transwell migration and invasion assays in addition to a wound-healing assay indicated that knockdown of ADAM12 inhibited PC-9 cell migration and invasion(Fig. 6D, E). Taken together, the abovepresented results suggested that ADAM12 expression was implicated in the metastasis of LUAD.

\section{Discussion}

Although currently available multimodal therapy has greatly improved the survival of LUAD patients, treatment outcomes remain unsatisfactory. Thus, the identification of novel therapeutic targets is of great value for LUAD diagnosis and treatment. ADAMs are members of a family of multidomain transmembrane and secretory proteins. Specific ADAMs are upregulated in human cancers and are associated with tumour progression and poor prognosis ${ }^{[29]}$. However, the role of ADAMs in LUAD has rarely been studied.

In the current work, we analysed TCGA data for the expression of 12 ADAMs with proteolytic enzyme activity in LUAD. ADAM12 was highly expressed in LUAD and significantly associated with poor prognosis. We then used the cBioPortal database to explore gene copy number variation underlying the abnormal expression of ADAM12. Further, we utilised the LinkedOmic database and GSEA to explore the potential biological functions of ADAM12, finding that ADAM12 was closely related to the TME. The TIMER and TISIDB resources were used to explore the impact of ADAM12 within the TIM. We constructed a prognostic model with ADAM12 and related immunomodulatory molecules. Finally, we verified the biological function of ADAM12through a number of in vitro assays. To the best of our knowledge, this is the first comprehensive analysis of ADAM12 in LUAD, and the first work to highlight the relationship of ADAM12 with the TIM.

An increasing body of evidence has established the abnormal expression of ADAMs during tumourigenesis ${ }^{[30,31]}$. ADAMs promoted tumour growth and metastasis by regulating the activation of growth factors (such as EGF), cytokines (TNF-a) and integrins ${ }^{[32]}$. Among the 12 ADAMs with proteolytic enzyme activity, ADAM9, 10 and 17 have been most extensively investigated within cancer. ADAM9 expression is typically high in cervical, ovarian, prostate, lung, pancreatic and other cancer types wherein it is associated with poor prognosis ${ }^{[12,33-36]}$. ADAM10 and ADAM17 are important a-secretases, which can cleave amyloid precursor protein into active fragments and were previously reported as related to poor breast cancer prognosis ${ }^{[37]}$. Studies have indicated that, compared with that in normal tissue, inflamed tissue, and benign tumour controls, ADAM10 expression was significantly increased in NSCLC cell lines and patient tumours. Further, it was associated with a poor prognosis ${ }^{[38]}$. Li et al. ${ }^{[5]}$ found that ADAM17 expression was higher in gastric cancer patients with lymph node metastasis than in those without. Further, ADAM17 was suggested to promote lymph node metastasis through Notch and Wnt signalling. In this study, we analysed ADAMs in 497 cases of LUAD and 54 normal lung tissues from TCGA database. We observed that the expression of most ADAMs was dysregulated in LUAD, with that of ADAM12 being significantly increased. 
ADAM12 expression was previously reported to be high in breast, oesophageal, pituitary tumor, and choriocarcinoma ${ }^{[16,17,39]}$. Huang et al. ${ }^{[16]}$ found that Inc015192 competitively binds to miR-34a through sponging activity, inhibiting ADAM12 expression as well as tumour cell invasion, metastasis and the epithelial-to-mesenchymal transition. Luo et al. ${ }^{[17]}$ reported that ADAM12 was highly expressed at the edge of oesophageal cancer tumour invasion, promoting the activation of focal adhesion kinase (FAK), which in turn increased ADAM12 expression through the FAK/JNK/C-Jun axis, forming a positive feedback loop. Therefore, targeting ADAM12 represents a promising strategy for the treatment of metastatic oesophageal cancer. In choriocarcinoma, ADAM12 silencing significantly increased the expression of autophagy-related proteins LC3B and ATG5, thereby promoting chorionic cell apoptosis, which indicated that ADAM12 may be a new therapeutic target for choriocarcinoma ${ }^{[39]}$. However, there have been limited studies on ADAM12 in NSCLC. Through bioinformatics analyses, Cheng et al. [19] previously reportedADAM12, BTK and ERG were mainly related to the activation of pathways involving focal adhesion and immune regulation within the TME. However, this is the only report on the biological function of ADAM12 in LUAD. We carried out in vitro experiments and observed, for the first time, that ADAM12 knockdown reduced the proliferation, migration and invasion of LUAD cells.

To further understand the biological role of ADAM12 in LUAD, 19,987 ADAM12 co-expressed genes were identified using the LinkedOmics database. KEGG enrichment analysis revealed that genes positively correlated with ADAM12 were enriched in ECM-receptor interactions and adhesion-related pathways, further confirming the association of ADAM12 with the TME. Qi et al. ${ }^{[40]}$ previously suggested that the ADAM family proteases could be used as potential biomarkers of immune infiltration in pancreatic cancer. Here, GSEA revealed that ADAM12 was related to B cell receptor, ECM receptor, $T$ cell receptor, and TOLL-like receptor signalling pathways as well as natural killer (NK) cell-mediated cytotoxicity, thus confirming the implication of ADAM12 within the TIM. Copy number variation describes the phenomenon of a DNA fragment with a difference in genecopy number, potentially leading changes in gene expression, which may in turn promote tumourigenesis. In this study, we found that ADAM12 mainly exhibited missense mutations, followed by deep deletions and amplifications. Interestingly, ADAM12 mRNA expression was highest in the cases of deep deletion. Further, arm-level gain was correlated with the infiltration of $\mathrm{B}$ cells, $\mathrm{CD} 4^{+} \mathrm{T}$ cells and dendritic cells. In addition, high amplification was associated with the tumour infiltration of $\mathrm{CD} 8^{+} \mathrm{T}$ cells and macrophages. In contrast, the degree of neutrophil infiltration was not affected by ADAM12 copy number alterations.

Immune markers are relevant for tumour prognosis and immunotherapy response prediction ${ }^{[41,42]}$. Zhang et al. ${ }^{[43}$ reported that deleterious $\mathrm{NOTCH}$ mutations may lead to the activation of immune-related genes and were associated with an improved tumour immunotherapy response, suggestive of their potential as predictive markers in NSCLC. Further, ZFHX3 mutations were also associated with enhanced immunogenicity and activation of anti-tumour immunity ${ }^{[44]}$, supporting their utility as immunotherapy predictive markers. In order to provide a theoretical basis for the relationship between ADAM12 and tumour immune infiltration, we determined the association between ADAM12 expression and various classic immune biomarkers. ADAM12 was strongly correlated with $\mathrm{CD} 4^{+}$cell markers BRIP1, CCNB1, 
EX01, KNTC1 and PRC1. Therefore, ADAM12 may be utilised as a biomarker of immune infiltration based on its putative relationship with these immune markers, thus predicting LUAD prognosis. Through the TISIDB database, we found that the immunostimulatory TNFSF4 and ADAM12 had a significant positive correlation, suggestive of ADAM12's implication in LUAD immunotherapy. We constructed a prognostic model based on ADAM12 and five related immunomodulatory molecule-encoding genes. The model accurately predicted patient prognosis. Based on the above results, we speculated that ADAM12expressionmayaffect the prognosis of LUAD patients through its effects within the TIM.

Nevertheless, our study had certain limitations. No in vitro or in vivo experiments were conducted to confirm the association of ADAM12 and immune infiltrations. Thus, further investigation is warranted in order to verify this relationship, allowing for a better understanding of ADAM12 in the context of tumour treatment.

\section{Conclusions}

In summary, we found that ADAM12 may act as an oncogene, affecting LUAD prognosis. Further, bioinformatics analysis suggested that ADAM12 and its co-expressed genes regulated ECM-receptor interactions and focal adhesions in LUAD. ADAM12 copy number variation was related to the level of immune cell infiltration, suggesting that it may play a role in regulating the TIM, which provides a new direction for future studies focused on LUAD biomarkers.

\section{List Of Abbreviations}

ADAM, a-disintegrin and metalloproteinase; LUAD, lung adenocarcinoma; KEGG, Kyoto Encyclopedia of Genes and Genomes; TIM, tumour immune microenvironment; NSCLC, non-small cell lung cancer; LUSC; lung squamous cell carcinoma; TNBC, triple-negative breast cancer; VEGFA, vascular endothelial growth factor A; ANGPT2, angiopoietin 2; PLAT, tissue plasminogen activator; TME, tumour microenvironment; MMP, matrix metalloproteinase; LASSO, Least Absolute Contraction and Selection Operator; FAK, focal adhesion kinase

\section{Declarations}

\section{Ethics approval and consent to participate}

Not applicable

\section{Consent for publication}

Not applicable

\section{Availability of data and materials}

All data generated or analyzed during this study are included in this published article 


\section{Competing interests}

The authors declare that they have no competing interests.Funding

This work was received no funding.

\section{Authors' contributions}

Conceived and designed the study strategy: JFP, YQX; Acquisition of data: statistical analysis and interpretation of data JFP, ZDH; Drafting or revision of the manuscript: JFP; Reference collection and data management: ZDH; Wrote the manuscript: JFP, YQX; Prepared the tables and fgures: ZDH; Study supervision: YQX; All authors read and approved the fnal manuscript.

\section{Acknowledgements}

The authors thank the TCGA database for providing high quality data.

\section{Authors' information}

${ }^{1}$ Department of Thoracic Oncology, Fujian Cancer Hospital, Fujian Medical University Cancer Hospital, Fuzhou 350014, China,

${ }^{2}$ Shengli Clinical Medical College of Fujian Medical University, Fuzhou 350001, China》

${ }^{3}$ Quanzhou First Hospital of Fujian Medical University, Quanzhou 362000, China

\section{References}

1. Bray F, Ferlay J, Soerjomataram I, Siegel RL, Torre LA, Jemal A. Global cancer statistics 2018 : GLOBOCAN estimates of incidence and mortality worldwide for 36 cancers in 185 countries. CA Cancer J Clin 2018, 68(6): 394-424.

2. Liu J, Cho SN, Akkanti B, Jin N, Mao J, Long W, Chen T, Zhang Y, Tang X, Wistub, II, Creighton CJ, Kheradmand F, DeMayo FJ. ErbB2 Pathway Activation upon Smad4 Loss Promotes Lung Tumor Growth and Metastasis. Cell Rep 2015, 10(9):1599-1613.

3. Osmani L, Askin F, Gabrielson E, Li QK. Current WHO guidelines and the critical role of immunohistochemical markers in the subclassification of non-small cell lung carcinoma (NSCLC): Moving from targeted therapy to immunotherapy. Semin Cancer Biol 2018, 52(Pt 1): 103-109.

4. Jones JC, Rustagi S, Dempsey PJ. ADAM Proteases and Gastrointestinal Function. Annu Rev Physiol 2016, 78: 243-276.

5. Li W, Wang D, Sun X, Zhang Y, Wang L, Suo J. ADAM17 promotes lymph node metastasis in gastric cancer via activation of the Notch and Wnt signaling pathways. Int J Mol Med 2019, 43(2): 914-926. 
6. Blobel CP. ADAMs: key components in EGFR signalling and development. Nat Rev Mol Cell Biol 2005, 6(1): 32-43.

7. Das SG, Romagnoli M, Mineva ND, Barille-Nion S, Jezequel P, Campone M, Sonenshein GE. miR-720 is a downstream target of an ADAM8-induced ERK signaling cascade that promotes the migratory and invasive phenotype of triple-negative breast cancer cells. Breast Cancer Res 2016, 18(1): 40.

8. Shen H, Li L, Zhou S, Yu D, Yang S, Chen X, Wang D, Zhong S, Zhao J, Tang J. The role of ADAM17 in tumorigenesis and progression of breast cancer. Tumour Biol 2016.

9. Yang B, Wang C, Xie H, Wang Y, Huang J, Rong Y, Zhang H, Kong H, Yang Y, Lu Y. MicroRNA-3163 targets ADAM-17 and enhances the sensitivity of hepatocellular carcinoma cells to molecular targeted agents. Cell Death Dis 2019, 10(10): 784.

10. Pelullo M, Nardozza F, Zema S, Quaranta R, Nicoletti C, Besharat ZM, Felli MP, Cerbelli B, d'Amati G, Palermo R, Capalbo C, Talora C, Di Marcotullio L, Giannini G, Checquolo S, Screpanti I, Bellavia D. Kras/ADAM17-Dependent Jag1-ICD Reverse Signaling Sustains Colorectal Cancer Progression and Chemoresistance. Cancer Res 2019, 79(21): 5575-5586.

11. Lu HY, Zu YX, Jiang XW, Sun XT, Liu TY, Li RL, Wu Q, Zhang YS, Zhao QC. Novel ADAM-17 inhibitor ZLDI-8 inhibits the proliferation and metastasis of chemo-resistant non-small-cell lung cancer by reversing Notch and epithelial mesenchymal transition in vitro and in vivo. Pharmacol Res 2019, 148: 104406.

12. Lin CY, Cho CF, Bai ST, Liu JP, Kuo TT, Wang LJ, Lin YS, Lin CC, Lai LC, Lu TP, Hsieh CY, Chu CN, Cheng DC, Sher YP. ADAM9 promotes lung cancer progression through vascular remodeling by VEGFA, ANGPT2, and PLAT. Sci Rep 2017, 7(1): 15108.

13. Wang Y, Lian YM, Ge CY. MiR-145 changes sensitivity of non-small cell lung cancer to gefitinib through targeting ADAM19. Eur Rev Med Pharmacol Sci 2019, 23(13): 5831-5839.

14. Mochizuki S, Shimoda M, Abe H, Miyamae Y, Kuramoto J, Aramaki-Hattori N, Ishii K, Ueno H, Miyakoshi A, Kojoh K, Okada Y. Selective Inhibition of ADAM28 Suppresses Lung Carcinoma Cell Growth and Metastasis. Mol Cancer Ther 2018, 17(11): 2427-2438.

15. Ota M, Mochizuki S, Shimoda M, Abe H, Miyamae $Y$, Ishii $K$, Kimura $H$, Okada Y. ADAM23 is downregulated in side population and suppresses lung metastasis of lung carcinoma cells. Cancer science 2016, 107(4): 433-443.

16. Huang X, Xie X, Liu P, Yang L, Chen B, Song C, Tang H, Xie X. Adam12 and Inc015192 act as ceRNAs in breast cancer by regulating miR-34a. Oncogene 2018, 37(49): 6316-6326.

17. Luo ML, Zhou Z, Sun L, Yu L, Sun L, Liu J, Yang Z, Ran Y, Yao Y, Hu H. An ADAM12 and FAK positive feedback loop amplifies the interaction signal of tumor cells with extracellular matrix to promote esophageal cancer metastasis. Cancer Lett 2018, 422: 118-128.

18. Wang J, Zhang Z, Li R, Mao F, Sun W, Chen J, Zhang H, Bartsch JW, Shu K, Lei T. ADAM12 induces EMT and promotes cell migration, invasion and proliferation in pituitary adenomas via EGFR/ERK signaling pathway. Biomed Pharmacother 2018, 97: 1066-1077. 
19. Yue $\mathrm{C}, \mathrm{Ma} \mathrm{H}$, Zhou Y. Identification of prognostic gene signature associated with microenvironment of lung adenocarcinoma. PeerJ 2019, 7: e8128.

20. Rossi GR, Trindade ES, Souza-Fonseca-Guimaraes F. Tumor Microenvironment-Associated Extracellular Matrix Components Regulate NK Cell Function. Front Immunol 2020, 11: 73.

21. Shiomi T, Lemaitre V, D'Armiento J, Okada Y. Matrix metalloproteinases, a disintegrin and metalloproteinases, and a disintegrin and metalloproteinases with thrombospondin motifs in nonneoplastic diseases. Pathol Int 2010, 60(7): 477-496.

22. Sternlicht MD, Werb Z. HOW MATRIX METALLOPROTEINASES REGULATE CELL BEHAVIOR. Annu Rev Cell Dev Biol 2001, 17: 463-516.

23. Groh V, Wu J, Yee C, Spies T. Tumour-derived soluble MIC ligands impair expression of NKG2D and Tcell activation. Nature 2002, 419(6908): 734-738.

24. Wu J, Mishra HK, Walcheck B. Role of ADAM17 as a regulatory checkpoint of CD16A in NK cells and as a potential target for cancer immunotherapy. Journal of leukocyte biology 2019, 105(6): 12971303.

25. Gao J, Aksoy BA, Dogrusoz U, Dresdner G, Gross B, Sumer SO, Sun Y, Jacobsen A, Sinha R, Larsson E, Cerami E, Sander C, Schultz N. Integrative analysis of complex cancer genomics and clinical profiles using the cBioPortal. Science signaling 2013, 6(269): pl1.

26. Vasaikar SV, Straub P, Wang J, Zhang B. LinkedOmics: analyzing multi-omics data within and across 32 cancer types. Nucleic Acids Res 2018, 46(D1): D956-d963.

27. Lu J, Huang XY, Wang YH, Xie JW, Wang JB, Lin JX, Chen QY, Cao LL, Li P, Huang CM, Zheng CH. POC1A acts as a promising prognostic biomarker associated with high tumor immune cell infiltration in gastric cancer. Aging 2020, 12(19): 18982-19011.

28. Ma C, Luo H, Cao J, Gao C, Fa X, Wang G. Independent prognostic implications of RRM2 in lung adenocarcinoma. J Cancer 2020, 11(23): 7009-7022.

29. Jiao X, Yu W, Qian J, Chen Y, Wei P, Fang W, Yu G. ADAM-17 is a poor prognostic indicator for patients with hilar cholangiocarcinoma and is regulated by FoxM1. BMC Cancer 2018, 18(1): 570.

30. Duffy MJ, McKiernan E, O'Donovan N, McGowan PM. Role of ADAMs in cancer formation and progression. Clin Cancer Res 2009, 15(4): 1140-1144.

31. Aydin D, Bilici A, Yavuzer D, Kefeli U, Tan A, Ercelep O, Mert A, Yuksel S, Ozcelik M, Isik D, Surmeli H, Odabasi $\mathrm{H}$, Aliustaoglu M. Prognostic significance of ADAM17 expression in patients with gastric cancer who underwent curative gastrectomy. Clinical \& translational oncology : official publication of the Federation of Spanish Oncology Societies and of the National Cancer Institute of Mexico 2015, 17(8): 604-611.

32. Mochizuki S, Okada Y. ADAMs in cancer cell proliferation and progression. Cancer science 2007, 98(5): 621-628.

33. Mohd Isa SA, Md Salleh MS, Ismail MP, Hairon SM. ADAM9 Expression in Uterine Cervical Cancer and Its Associated Factors. Asian Pacific journal of cancer prevention : APJCP 2019, 20(4): 1081-1087. 
34. Guo T, Yuan D, Lin M, Zhu D, Xu N, Wang J. Aberrant expression of ADAM9 in ovarian cancer and its clinical significance. Journal of clinical laboratory analysis 2020, 34(4): e23136.

35. Hua Y, Liang C, Miao C, Wang S, Su S, Shao P, Liu B, Bao M, Zhu J, Xu A, Zhang J, Li J, Wang Z. MicroRNA-126 inhibits proliferation and metastasis in prostate cancer via regulation of ADAM9. Oncol Lett 2018, 15(6): 9051-9060.

36. Xing C, Ye H, Wang W, Sun M, Zhang J, Zhao Z, Jiang G. Circular RNA ADAM9 facilitates the malignant behaviours of pancreatic cancer by sponging miR-217 and upregulating PRSS3 expression. Artificial cells, nanomedicine, and biotechnology 2019, 47(1):3920-3928.

37. Tsang JYS, Lee MA, Chan TH, Li J, Ni YB, Shao Y, Chan SK, Cheungc SY, Lau KF, Tse GMK. Proteolytic cleavage of amyloid precursor protein by ADAM10 mediates proliferation and migration in breast cancer. EBioMedicine 2018, 38: 89-99.

38. Yoneyama T, Gorry M, Sobo-Vujanovic A, Lin Y, Vujanovic L, Gaither-Davis A, Moss ML, Miller MA, Griffith LG, Lauffenburger DA, Stabile LP, Herman J, Vujanovic NL. ADAM10 Sheddase Activity is a Potential Lung-Cancer Biomarker. J Cancer 2018, 9(14): 2559-2570.

39. Wang L, Tan Z, Zhang Y, Kady Keita N, Liu H, Zhang Y. ADAM12 silencing promotes cellular apoptosis by activating autophagy in choriocarcinoma cells. Int J Oncol 2020, 56(5): 1162-1174.

40. Qi B, Liu H, Dong Y, Shi X, Zhou Q, Zeng F, Bao N, Li Q, Yuan Y, Yao L, Xia S. The nine ADAMs family members serve as potential biomarkers for immune infiltration in pancreatic adenocarcinoma. Peer $J$ 2020, 8: e9736.

41. Goodman A, Patel SP, Kurzrock R. PD-1-PD-L1 immune-checkpoint blockade in B-cell lymphomas. Nature reviews Clinical oncology 2017, 14(4): 203-220.

42. Kim S, Koh J, Kim MY, Kwon D, Go H, Kim YA, Jeon YK, Chung DH. PD-L1 expression is associated with epithelial-to-mesenchymal transition in adenocarcinoma of the lung. Human pathology 2016, 58: 7-14.

43. Zhang K, Hong X, Song Z, Xu Y, Li C, Wang G, Zhang Y, Zhao X, Zhao Z, Zhao J, Huang M, Huang D, Qi C, Gao C, Cai S, Gu F, Hu Y, Xu C, Wang W, Lou Z, Zhang Y, Liu L. Identification of Deleterious NOTCH Mutation as Novel Predictor to Efficacious Immunotherapy in NSCLC. Clin Cancer Res 2020, 26(14):3649-3661.

44. Zhang J, Zhou N, Lin A, Luo P, Chen X, Deng H, Kang S, Guo L, Zhu W, Zhang J. ZFHX3 mutation as a protective biomarker for immune checkpoint blockade in non-small cell lung cancer. Cancer immunology, immunotherapy : CII 2021, 70(1):137-151.

\section{Table}

Table1 Difference of ADAMs Expression Between Lung Adenocarcinoma Tissues and Adjacent Normal Tissues 


\begin{tabular}{|c|c|c|c|c|}
\hline gene & conMean & treatMean & $\log \mathrm{FC}$ & pValue \\
\hline ADAM12 & 0.32386449 & 3.295096519 & 3.346858492 & $7.62 \mathrm{E}-23$ \\
\hline ADAM2 2 & 0.79951090 & 3.985412053 & 2.31753927 & $2.97 \mathrm{E}-22$ \\
\hline ADAM8 & 3.25545584 & 15.45727836 & 2.247354842 & $5.03 \mathrm{E}-22$ \\
\hline ADAM15 & 17.6864443 & 37.22162169 & 1.073496872 & $5.88 \mathrm{E}-19$ \\
\hline ADAM33 & 1.00722757 & 0.520454364 & -0.952546108 & $4.35 \mathrm{E}-16$ \\
\hline ADAM1s & 2.35254998 & .505089374 & 30353 & 2.99E-07 \\
\hline ADAM9 & 21.9597104 & 35.41641168 & 0.689559019 & $3.82 \mathrm{E}-05$ \\
\hline \multicolumn{5}{|c|}{ ADAM1 78.9087297168 .251008544} \\
\hline \multicolumn{5}{|c|}{ ADAM10 8.4333860098.014731621-0.073457779 0.002176884 } \\
\hline \multicolumn{5}{|c|}{ ADAM20 0.0321279630.097571876 1.6026358720 .009902691} \\
\hline \multicolumn{5}{|c|}{ ADAM30 0.0005627380.003890081 2.7892660180.375624568 } \\
\hline \multicolumn{5}{|c|}{ ADAM21 0.0543666440 .1162358571 .0962615090 .612657416} \\
\hline
\end{tabular}

\section{Figures}


A

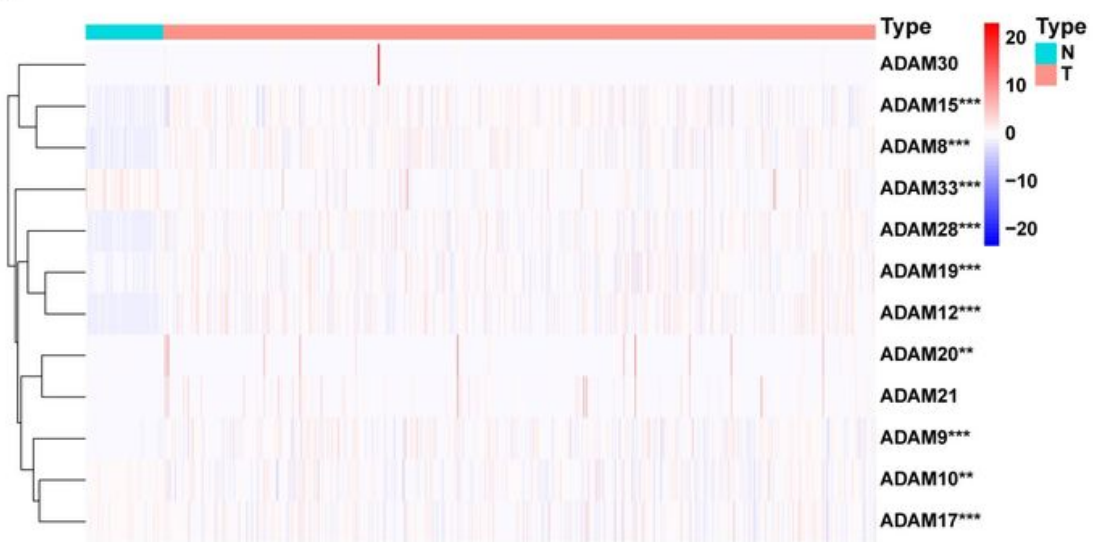

B

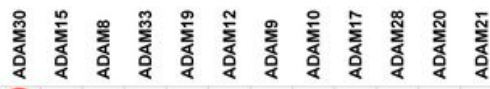

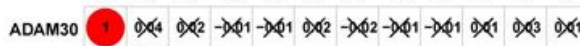

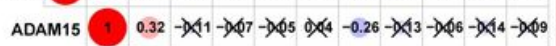

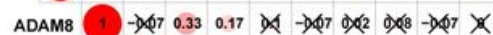

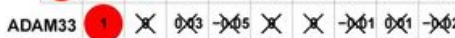

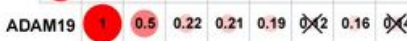
$\begin{array}{llllllll}\text { ADAM12 (1) } 0.16 & 0.32 & 0.26 & \times(2 & 0.18 & 0.21\end{array}$ ADAM9 (1) $0.33 \quad 0.26 \quad 0.17 \quad D \times 6 \quad D \times 6$ $\begin{array}{llllll}\text { ADAM10 (1) } & 0.48 & 0.3 & 0.41 & 0.35\end{array}$ ADAM17 (1) $\$ 10.26 \quad 0.34$ ADAM28 (1) 0.27 D ADAM 20 (1) 0.6 ADAM21 (1)

C

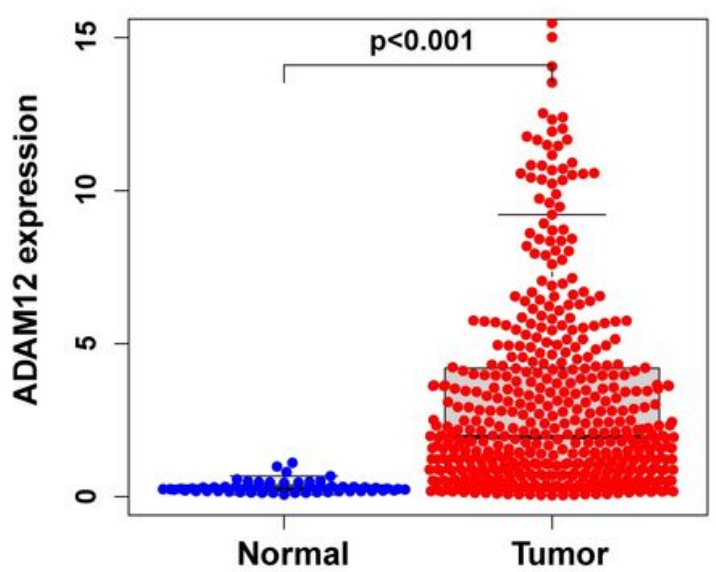

D

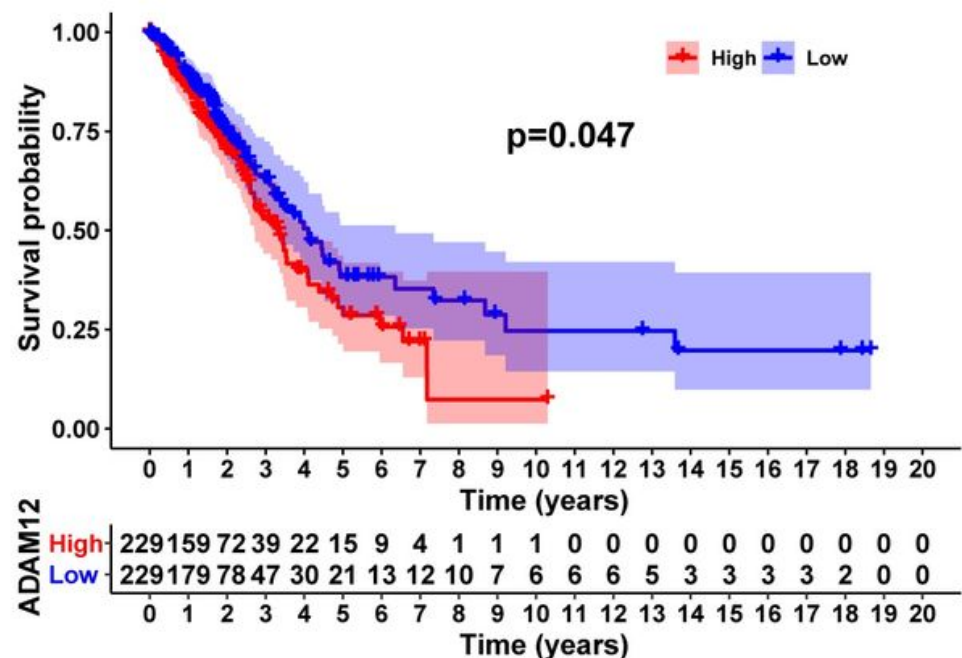

Figure 1

Differences in the expression of ADAMs between lung adenocarcinoma (LUAD) and healthy tissues in TCGA database. (A) heatmaps were used to visualise the differential expression levels of ADAMs between tumour and healthy tissue; (B) Spearman correlation analysis of the 12 ADAMs; (C) differential expression levels of ADAM12 between LUAD tissues and healthy tissues; (D) prognostic analysis of ADAM12 in high and low expression groups. N, normal sample; T, tumour sample; * $p<0.05, * * p<0.01, * *$ * $p<0.001$. 
A

Study of origin

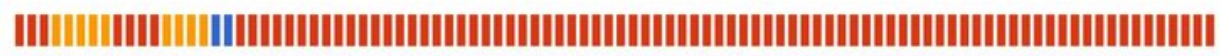

Profiled for copy number alterations

Profiled for mutations

ADAM12

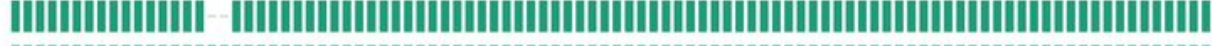

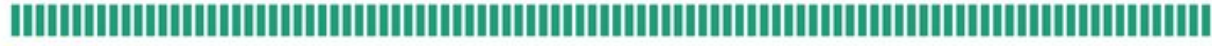

Genetic Alteration

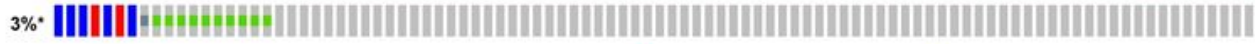

Study of origin

|| Missense Mutation (unknown significance)

| Amplification (unknown significance)

Deep Deletion (unknown significance)

Profiled for cops

alterations

Profiled for mutations

B

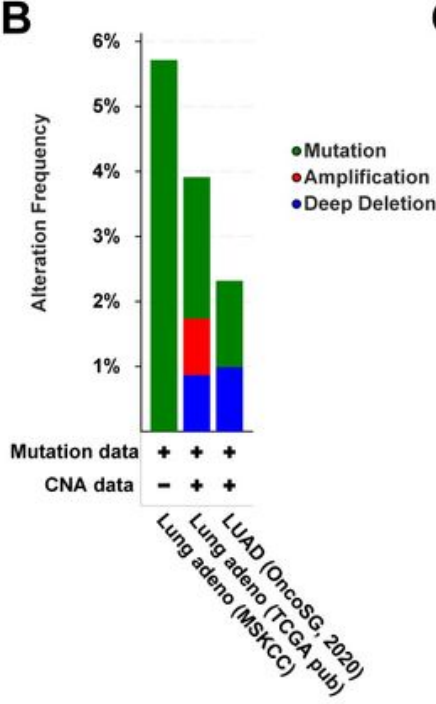

$\mathbf{E}$

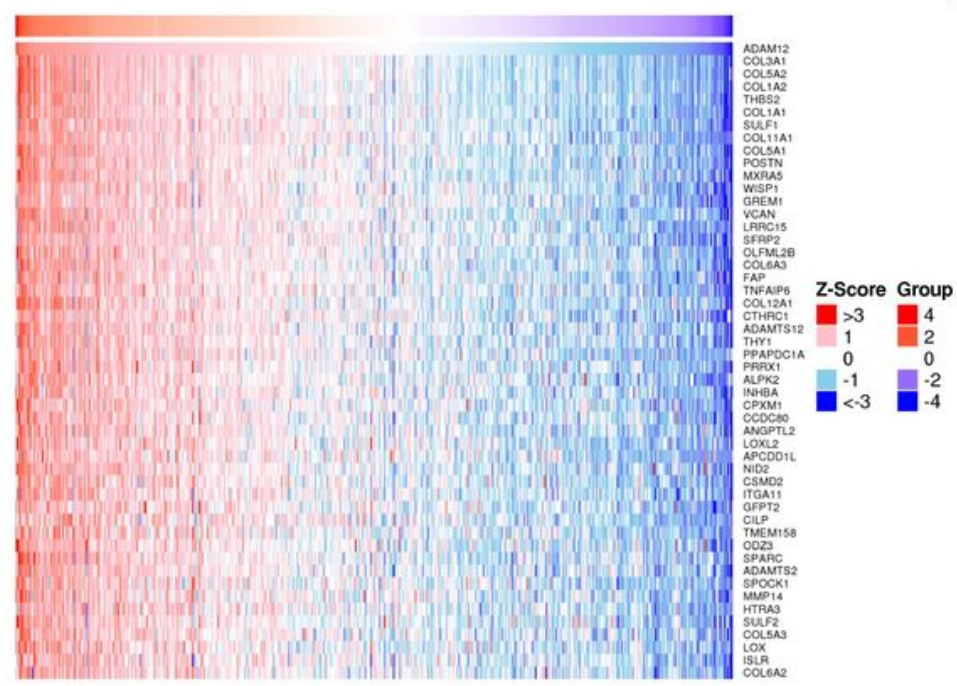

C ADAM12: Putative copy-number alterations from GISTIC

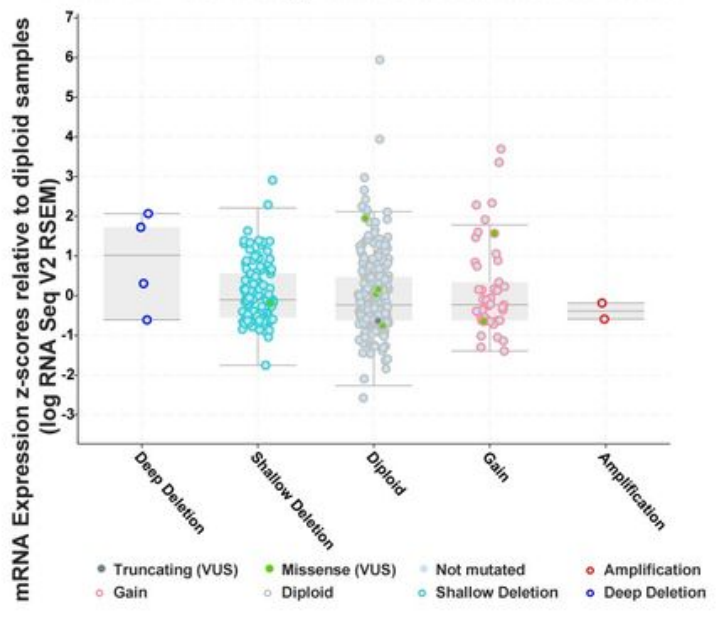

D

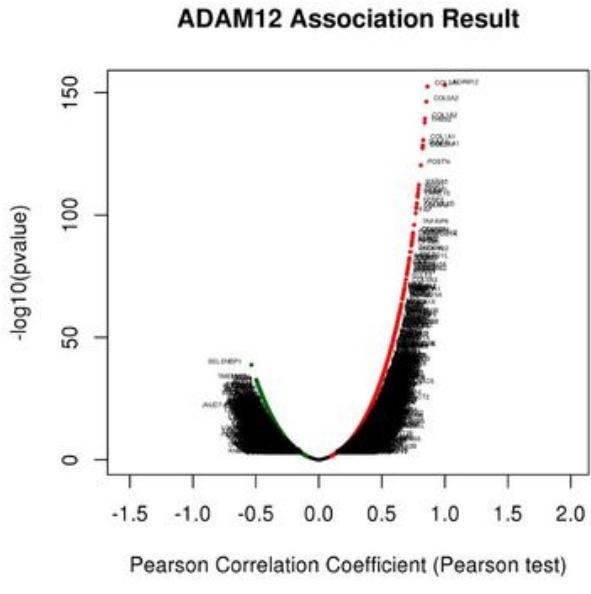

$\mathbf{F}$

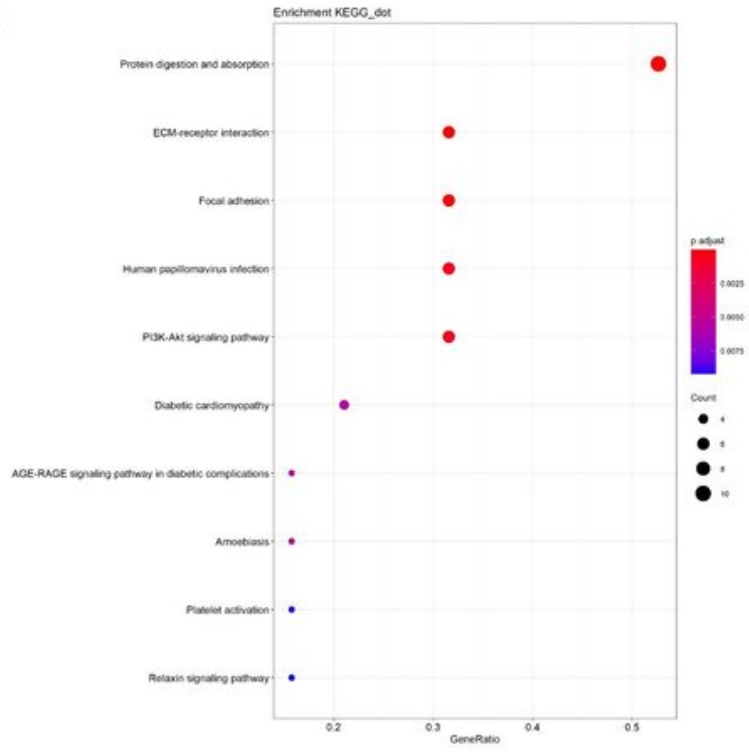

\section{Figure 2}

ADAM12 co-expressed genes and ADAM12 copy number variation lead to changes in expression. (A, B, C) ADAM12 genetic alterations mainly included missense mutations, amplifications and deep deletions. The highestADAM12 mRNA expression was observed in cases of deep deletions; (D, E) LinkedOmics database analysis of ADAM12 co-expressed genes and strong positive correlation of ADAM12 in LUAD; (F) KEGG pathway enrichment analysis of ADAM12 and positively correlated genes. 
A

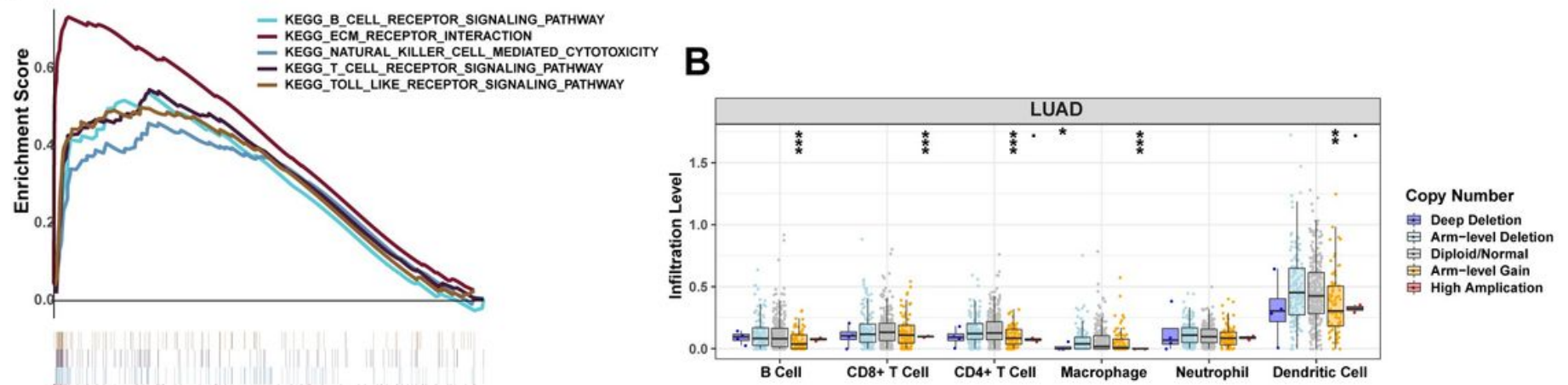

C

High expression <-----------> Low expression

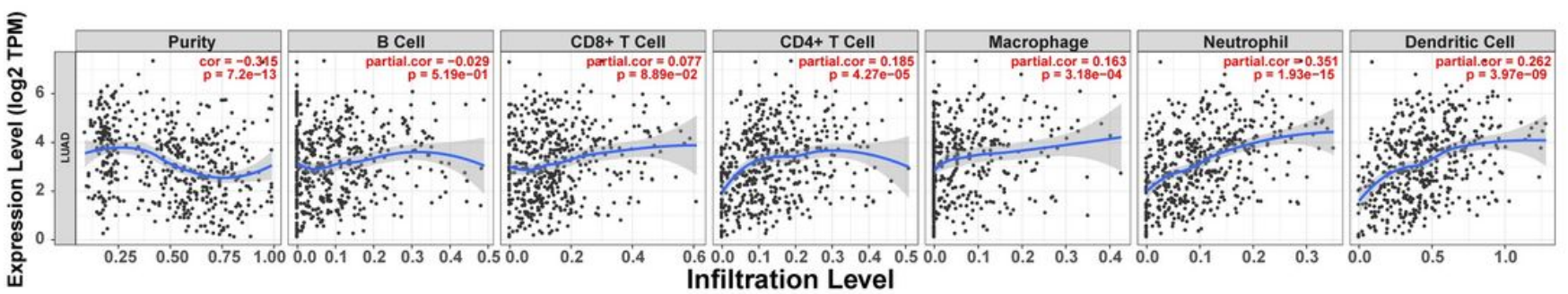

D

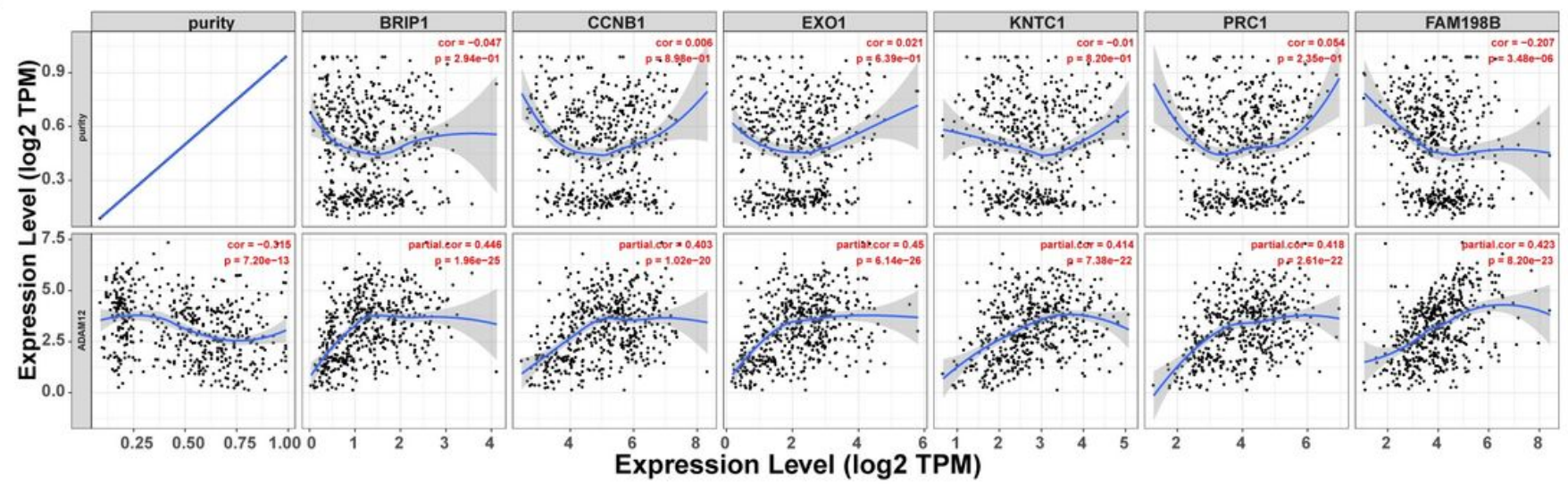

\section{Figure 3}

Influence of ADAM12 on the tumour immune microenvironment(TIM). (A) Gene set enrichment analysis for signalling pathways related to the differential expression of ADAM12 (FDR<0.05, NOM p-value $<0.05$ ); (B) ADAM12 copy number changes were correlated with the degree of immune cell infiltration based on TIMER analysis; (C) The expression of ADAM12 was positively correlated with the degree of infiltration of most immune cell types; (D) The correlation between immune cell markers expression and ADAM12. 
A

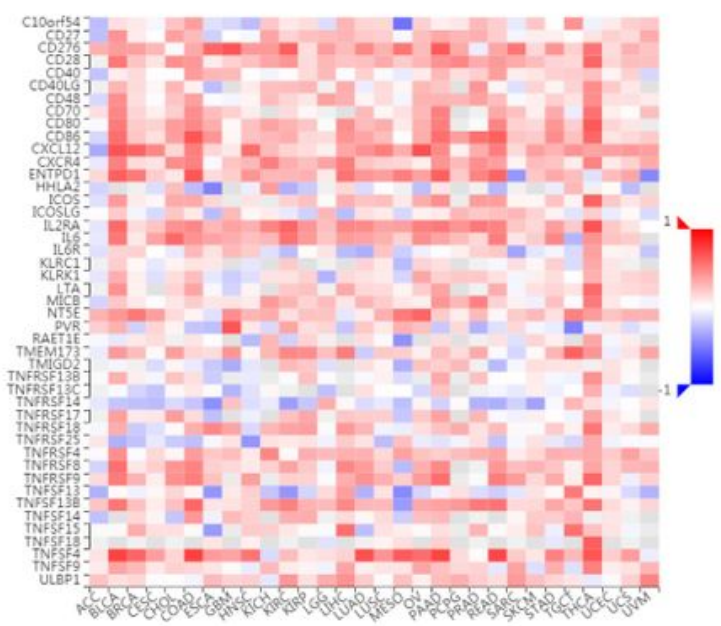

B
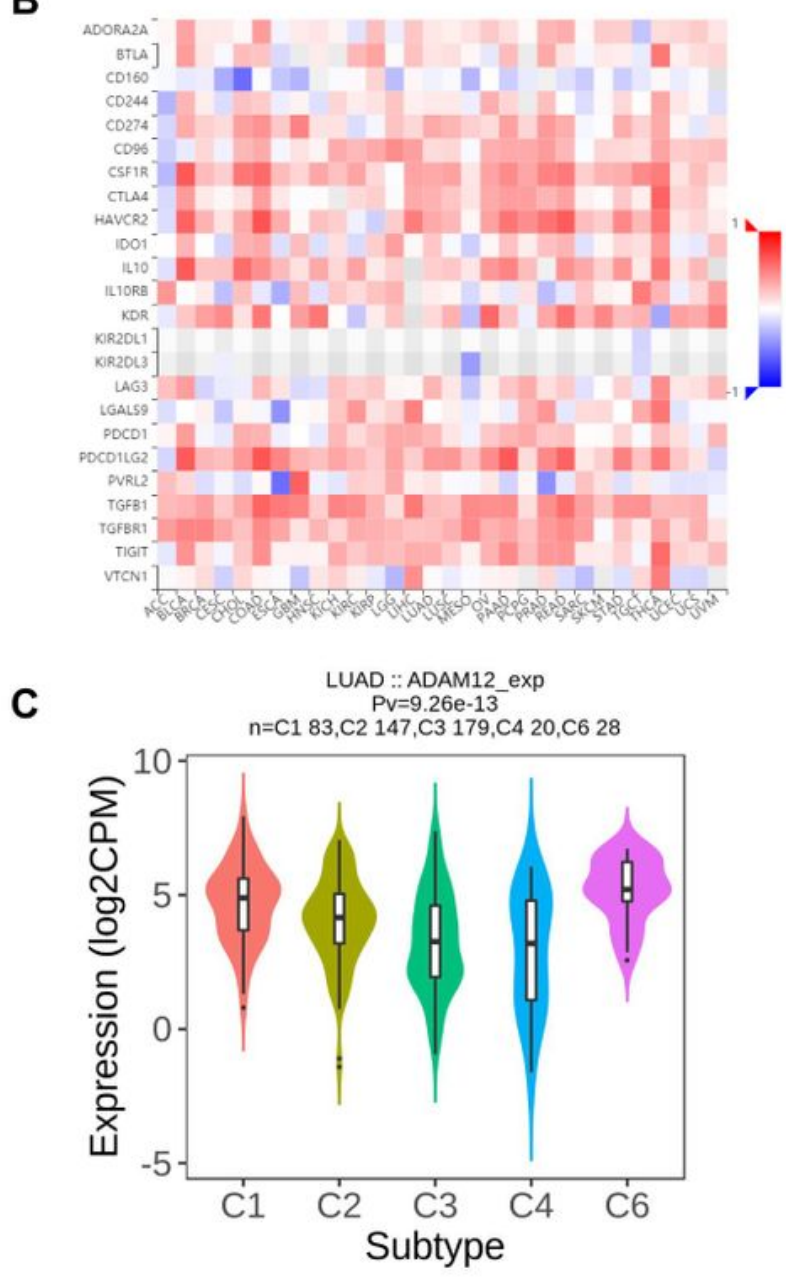
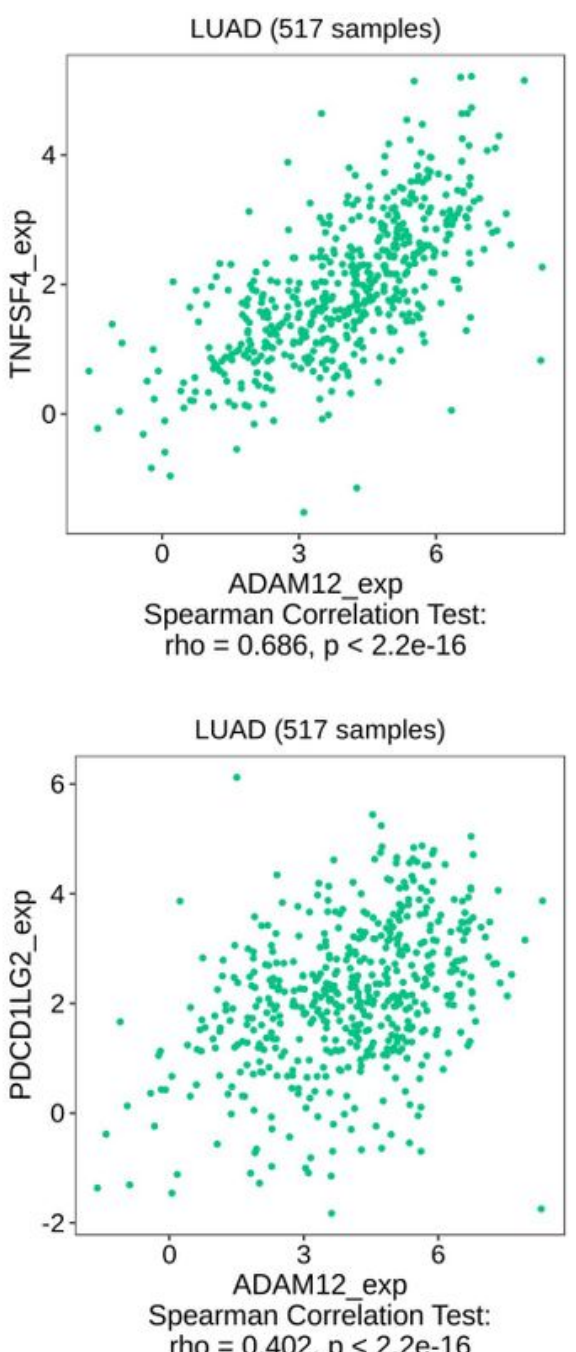

\section{Figure 4}

ADAM12-related immunomodulatory molecules. (A, B) ADAM12-related immunostimulatory and immunoinhibitory proteins; (C) ADAM12 expression in different immune subtypes of LUAD. 
A
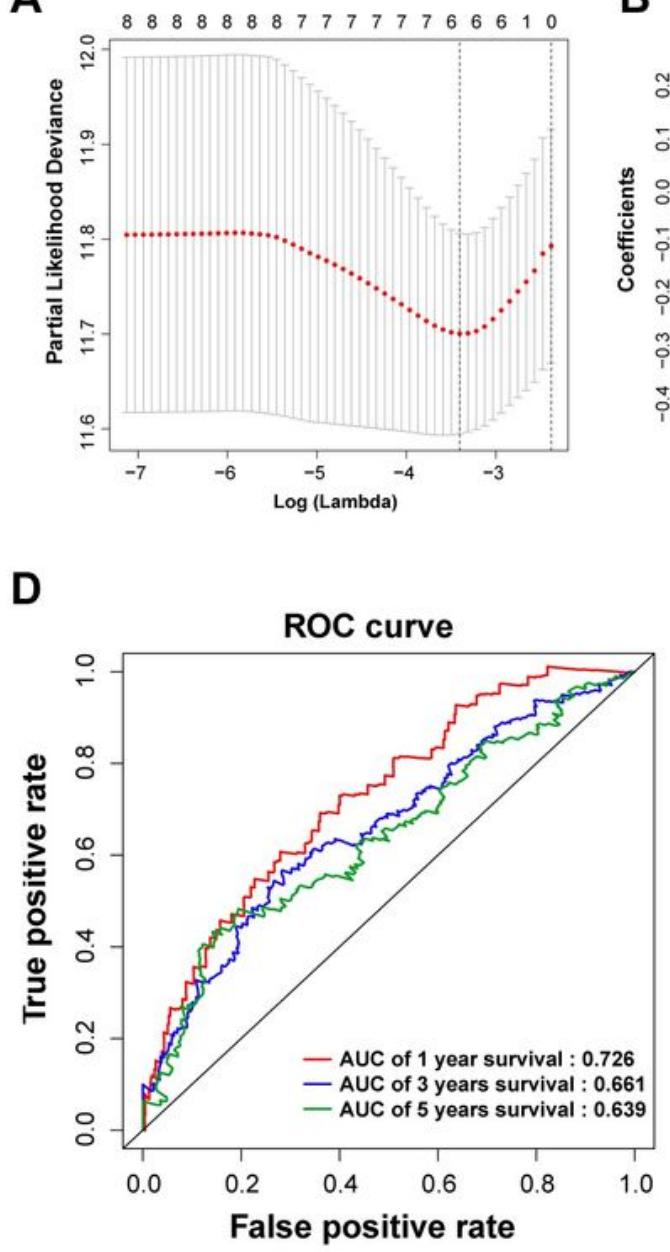

B

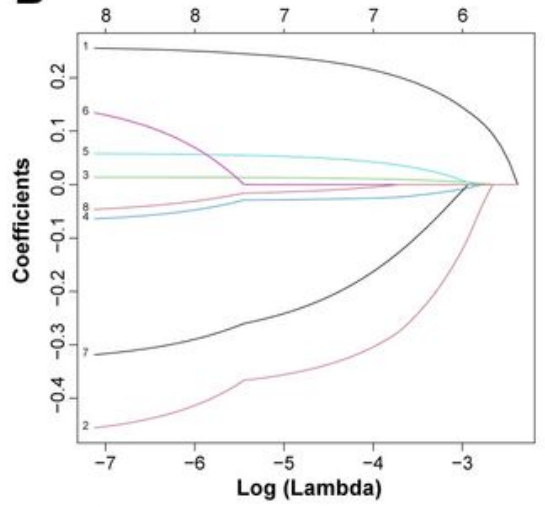

E
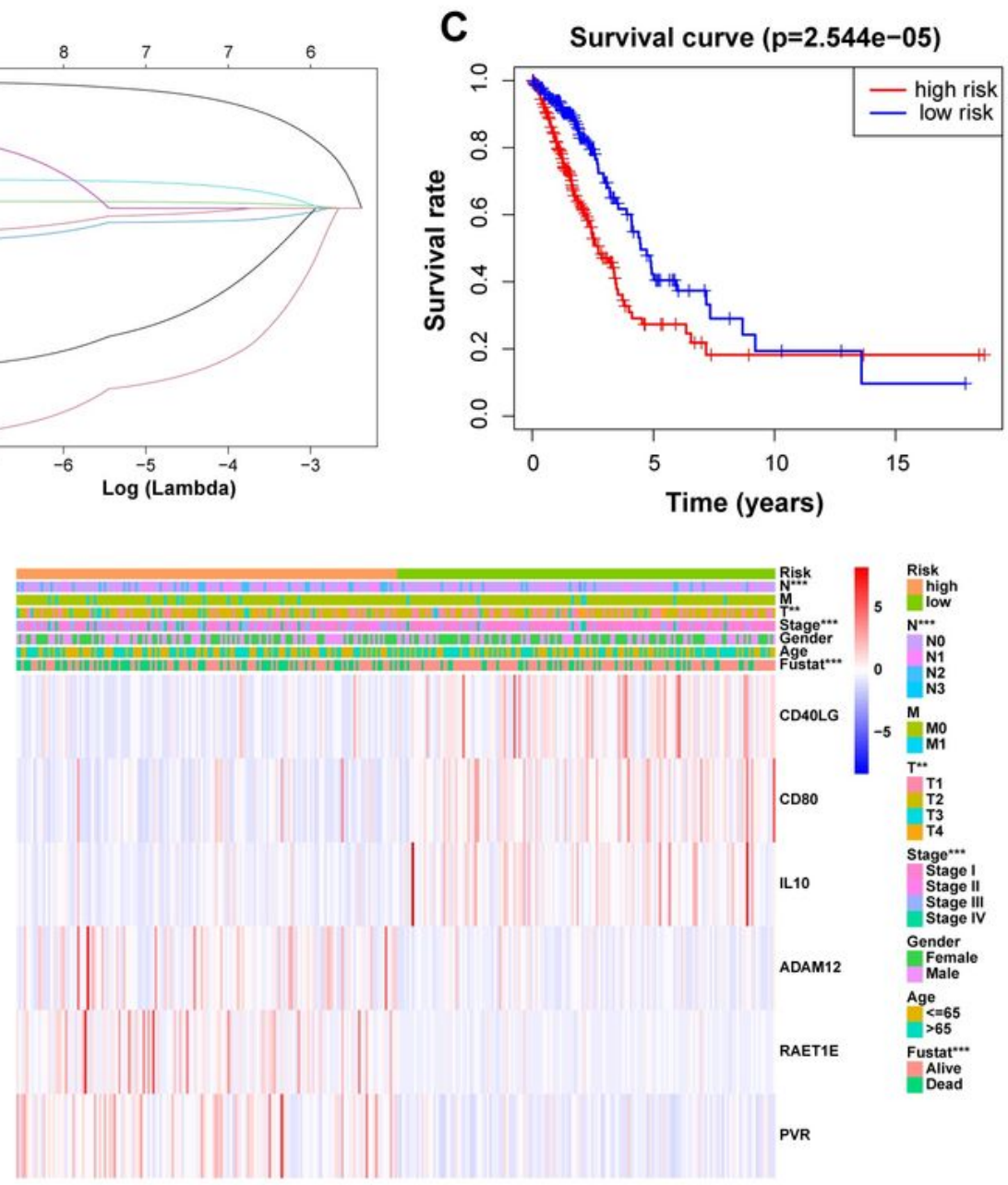

$\mathbf{F}$

p Value Hazard ratio

G

p Value Hazard ratio
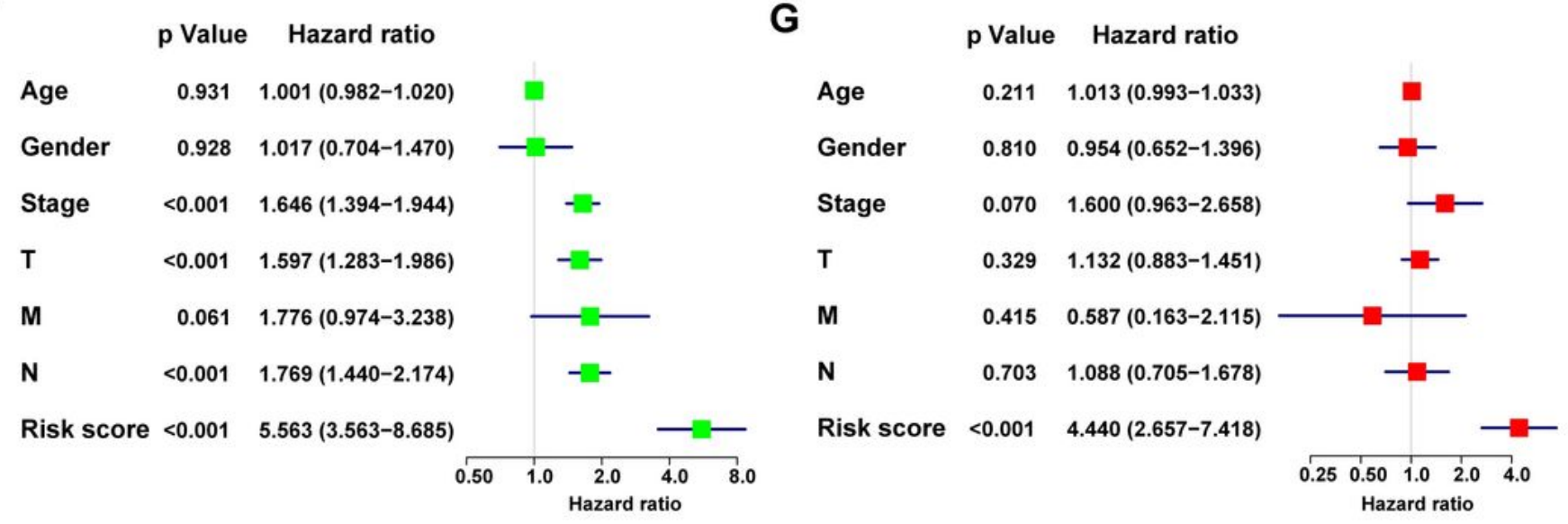

\section{Figure 5}

A prognostic model based on ADAM12 and related immunomodulatory molecules. (A, B) Least Absolute Contraction and Selection Operator (LASSO) algorithm was employed to obtain the coefficients of genes selected in order to assess their prognostic merit; (C) Kaplan-Meier overall survival curve of LUAD patients; (D) 1-, 3-, and 5-year ROC curves showing the predictive accuracy of the prognostic model in LUAD patients; (E) The relationship between risk score and clinicopathological characteristics of LUAD 
patients; (F) Univariate Cox regression analysis indicated that grade, $\mathrm{T}$ stage, $\mathrm{N}$ stage, and risk score were significantly related to overall survival; $(\mathrm{G})$ Multivariate Cox regression analysis further suggested that risk score was an independent diagnostic indicator of LUAD. ${ }^{*} p<0.05,{ }^{* \star} p<0.001,{ }^{\star \star \star} p<0.0001$.

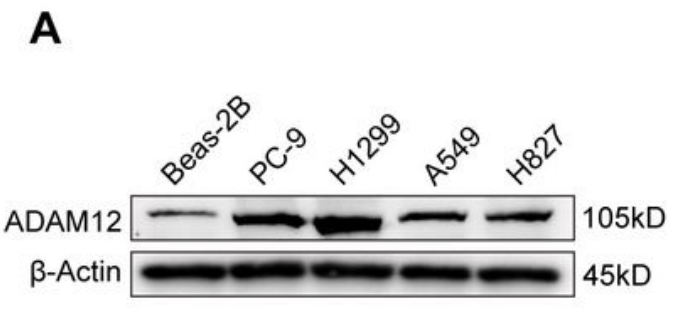

C
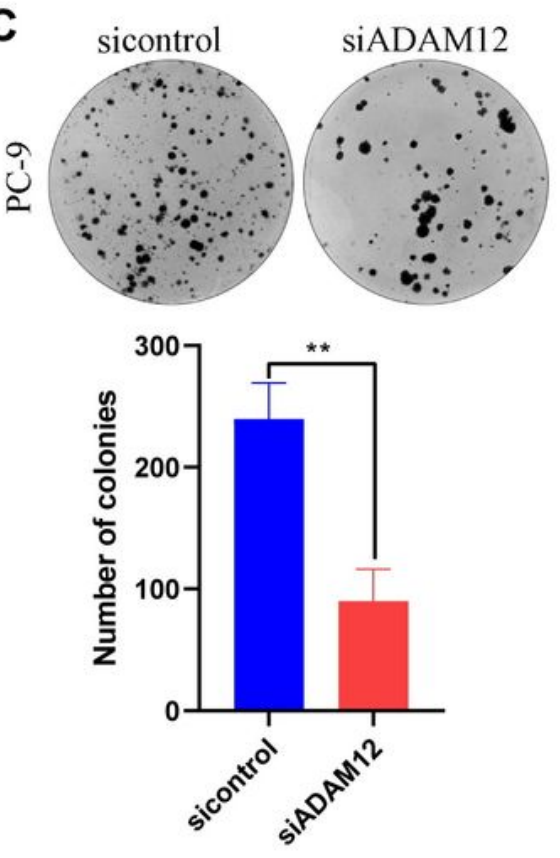

E

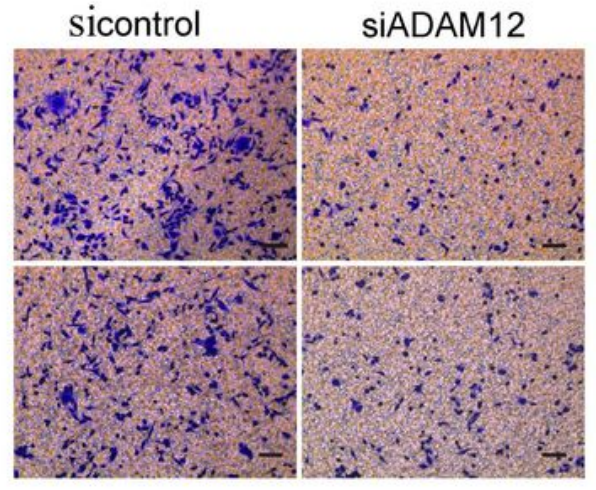

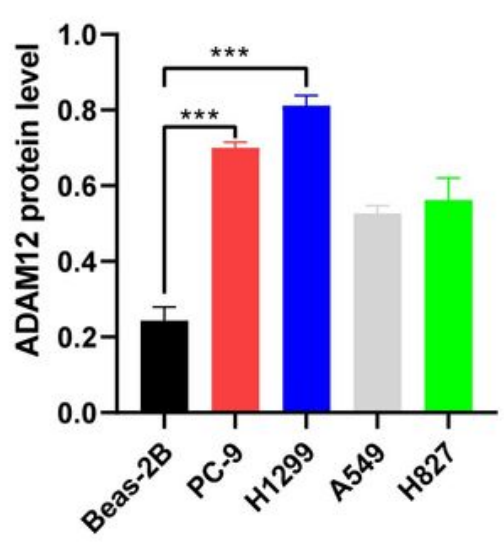

B
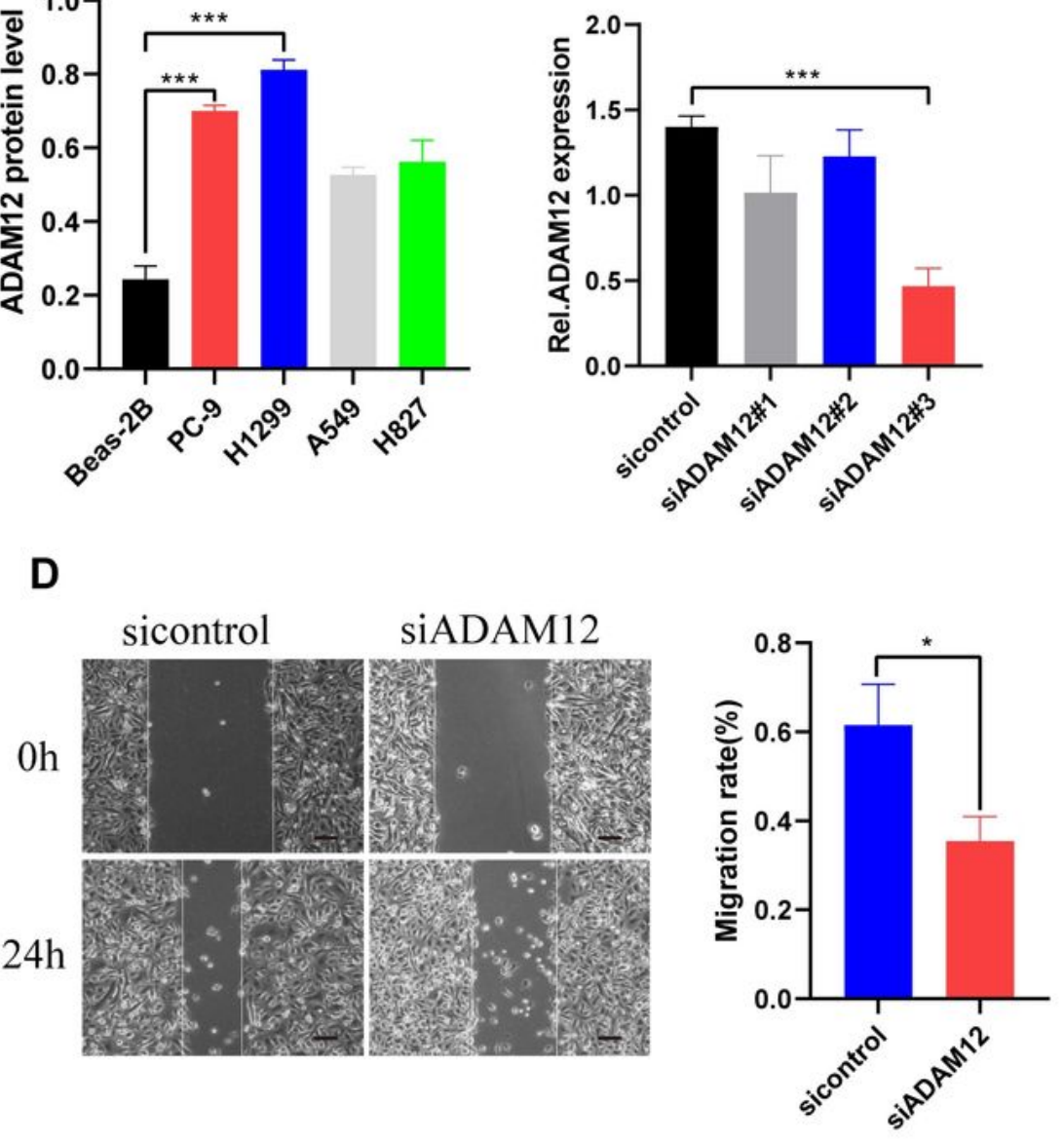

Figure 6

Knockdown of ADAM12 inhibited the growth and metastasis of LUAD cells in vitro. (A) Western blotting experiment to detect the expression of ADAM12 in BEAS-2B and various LUAD cells; (B) siADAM12 was 
transfected into PC-9 cells. siADAM12\#3 had the highest knockdown efficiency; (C) Cell colony formation assay for the assessment of cell proliferation ability. After knockdown of ADAM12, cell proliferation ability was significantly suppressed; Scale bars, 100um. (D, E) Transwell migration and wound-healing assays indicated that knockdown of ADAM12 inhibited PC-9 cell migration and invasion; Scale bars, 100um. ${ }^{*} p<0.05,{ }^{* \star} p<0.001,{ }^{* \star *} p<0.0001$.

\section{Supplementary Files}

This is a list of supplementary files associated with this preprint. Click to download.

- Additionalfle1.docx

- Additionalfle2.docx 